\title{
Synthesis of Fully Functionalized 3-Bromoazaspiro[4.5]trienones through Ugi Four-Component Reaction (Ugi-4CR) followed by ipso-Bromocyclization
}

\author{
Saeed Balalaie ${ }^{* a, b}$ (i) \\ Hadiseh Bakhshaei Ghoroghaghaei ${ }^{a}$ \\ Nahid S. Alavijeh ${ }^{\mathrm{a}}$ \\ Fatemeh Darvish ${ }^{\mathrm{a}}$ \\ Frank Rominger ${ }^{c}$ \\ Hamid Reza Bijanzadeh ${ }^{d}$ \\ a Peptide Chemistry Research Center, K. N. Toosi Universi- \\ ty of Technology, P. O. Box 15875-4416, Tehran, Iran \\ balalaie@kntu.ac.ir \\ b Medical Biology Research Center, Kermanshah University \\ of Medical Sciences, Kermanshah, Iran \\ ' Organisch-Chemisches Institut der Universitaet Heidel- \\ berg, Im Neuenheimer Feld 270, 69120 Heidelberg, Ger- \\ many \\ ${ }^{d}$ Department of Environmental Sciences, Faculty of Natu- \\ ral Resources and Marine Sciences, Tarbiat Modares Uni- \\ versity, Tehran, Iran
}

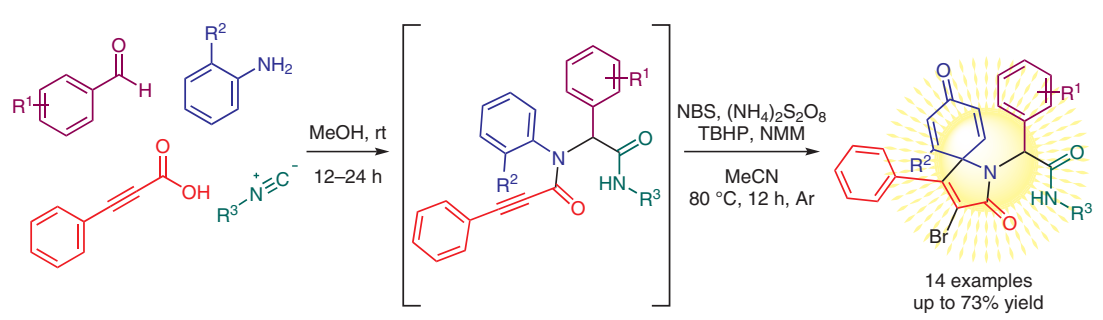

Received: 03.05.2018

Accepted after revision: 15.06.2018

Published online: 19.07 .2018

DOI: 10.1055/s-0037-1610205; Art ID: so-2018-d0033-I

License terms: cc)

Abstract Biologically attractive azaspiro[4.5]trienones have been prepared via Ugi four-component reaction (Ugi-4CR) followed by brominemediated ipso-cyclization. This allows a straightforward synthetic route to a diverse collection of fully functionalized 3-bromoazaspiro[4,5]trienones in moderate to good yields that can be used as templates for further modifications.

Key words alkynes, spiro compounds, cyclization, radical reaction, ring closure

Azaspirocycles, which are nitrogen-containing spirocyclic scaffolds, play significant roles in synthetic and medicinal chemistry. Literature searches on azaspirocyclic scaffolds have shown that these derivatives possess a broad spectrum of biological and pharmacological properties such as antimitotic, cytotoxic, antibacterial, antimicrobial, ant-inflammatory, antioxidative and antidepressant activities. ${ }^{1}$ A few examples of natural and synthetic drugs containing an azaspirocyclic skeleton are shown in Figure $1 .^{2}$ Consequently, great efforts have been made to synthesize diverse azaspirocycles libraries to facilitate the incorporation of these moieties into more biologically and pharmaceutically active molecules. ${ }^{3-7}$

Among the recently synthesized azaspirocycle-containing compounds, azaspiro[4.5]trienones have attracted a great deal of attention due to their chemistry and their biological activities. ${ }^{8}$ Very recently, and in the light of the intense interest to develop constrained tamoxifen mimics,
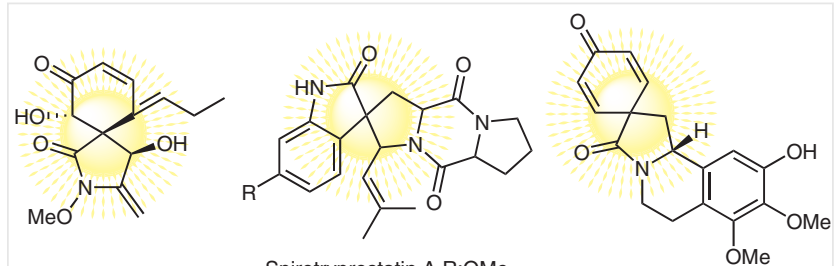

Spirostaphylotrichin A

Spirotryprostatin A R:OMe Spirotryprostatin B R:H

Annosqualine
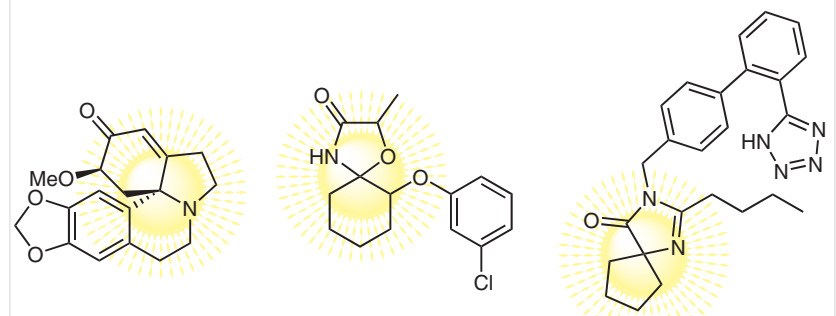

(+) - Erythratinone

Enilospirone

Irbesartan

Figure 1 Structures of some natural and synthetic drugs containing an azaspirocyclic skeleton

Srivastava et al. reported azaspiro[4,5]trienones as novel scaffolds for anticancer drug development (Figure 2, top structure). ${ }^{9}$ Furthermore, these compounds serve as valuable intermediates for the construction of azaspiro-fused tricyclic cores with promising anticancer activity by inducing DNA damage (Figure 2). ${ }^{10-12}$ Therefore, more attention has been drawn to the synthesis of functionalized azaspiro[4.5]trienones suitable for further derivatization processes. $^{13-15}$ 


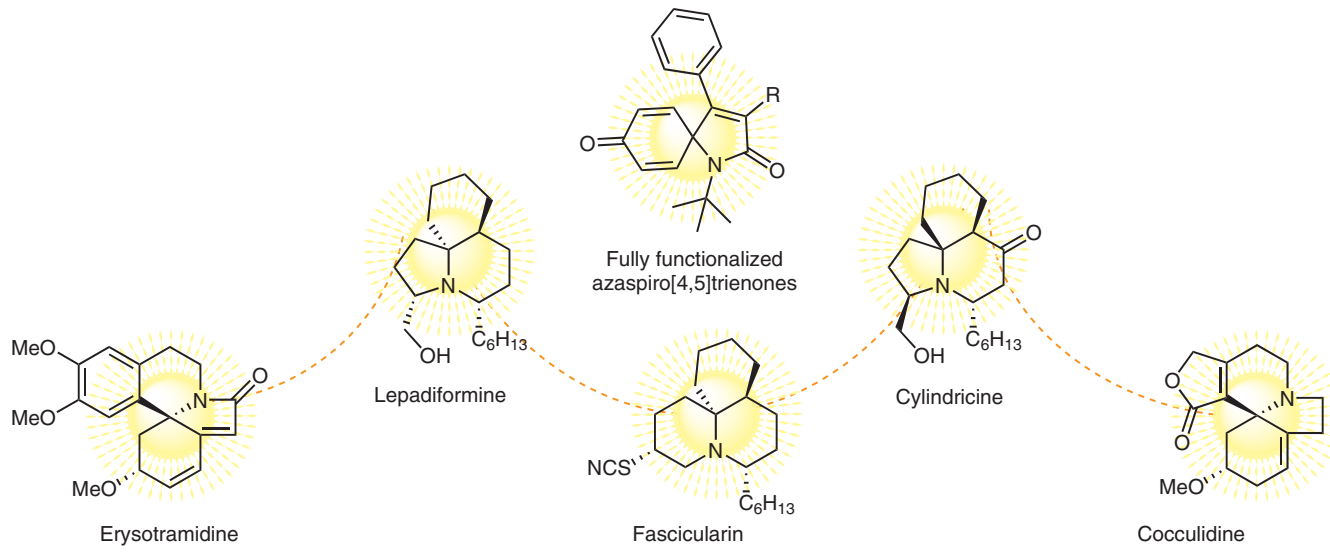

Figure 2 Recently reported cytotoxic azaspiro[4.5]trienones and cytotoxic alkaloids with azaspiro-fused tricyclic cores

Brominated substrates are excellent synthons for further functionalization because they can be further used in well-established cross-coupling reactions, whereas other approaches to such transformations are complex and often result in significant by-product formation. ${ }^{16}$ In this respect, especially in light of the fact that brominated triene-diones are ideal scaffolds for further elaboration in the diversityoriented synthesis, Qiu et al. have recently described the synthesis of 3-bromo-1-azaspiro[4.5]deca-3,6,9-triene-2,8dione through a novel $\mathrm{ZnBr}_{2}$-promoted oxidative ipso-annulation of $\mathrm{N}$-arylpropiolamide. ${ }^{5}$

In light of these findings and as a result of our interest in combination of the Ugi four-component reaction (Ugi-4CR) with efficient post-transformations for generating complex and diverse molecular libraries, ${ }^{17}$ we wish to report herein, a simple procedure for the synthesis of fully functionalized 3-bromoazaspiro[4.5]trienones via Ugi-4CR followed by ipso-bromocyclization. (Scheme 1).

Ugi 4-CR of 4-methoxybenzaldehyde (1a), aniline (2a), phenylpropiolic acid (3), and tert-butyl isocyanide (4a) in methanol at room temperature furnished the corresponding Ugi adduct $\mathbf{5 a}$ in $83 \%$ yield. This compound was chosen as the model substrate to investigate the bromine-mediated ipso-cyclization conditions. Application of $\left(\mathrm{NH}_{4}\right)_{2} \mathrm{~S}_{2} \mathrm{O}_{8} /$ TBHP ( 3 equiv/5 equiv) as the oxidant in the presence of $\mathrm{N}$-methylmorpholine (NMM, 0.5 equiv) in acetonitrile at $80^{\circ} \mathrm{C}$ un- der argon atmosphere produced the desired product $\mathbf{6 a}$ with a yield of $88 \%$. After screening solvents under these conditions, acetonitrile was found to be the best solvent (Table 1, entries 1-7). When $N$-methyl-2-pyrrolidone (NMP) was used in place of NMM, a marginal decrease in yield was recorded (entry 8). The application of NMM gave 6a with a yield of $88 \%$, while replacement with triethylamine (TEA), 1,8-diazabicyclo[5.4.0]undec-7-ene (DBU) and $\mathrm{N}, \mathrm{N}$-diisopropylethylamine (DIEA) resulted in lower yields (entries 9-11). Other radical initiators including di-tert-butyl peroxide (DTBP), benzoyl peroxide (BP), and $m$-chloroperoxybenzoic acid ( $m$-CPBA) were then employed, but all failed to give satisfactory yields (entries 12-14).

After carrying out the Ugi 4-CR, the solvent was evaporated under reduced pressure, and the conditions were switched for the bromine-mediated ipso-cyclization, which gave compound $\mathbf{6 a}$ in $67 \%$ overall yield, comparable with the $73 \%$ total yield obtained in the two-step procedure. Subsequently, the scope of the reaction was investigated by using different aromatic aldehydes, anilines, and isocyanides (Scheme 2). ${ }^{18}$

The structures of the products were confirmed based on NMR spectroscopy and HRMS (ESI) analysis. The characteristic resonances in the ${ }^{1} \mathrm{H}$ NMR spectra of all synthesized compounds appeared as four double doublets with coupling constants 9.9 and $1.8 \mathrm{~Hz}$ for the $\mathrm{sp}^{2} \mathrm{C}-\mathrm{H}$. The ${ }^{13} \mathrm{C}$ NMR spec-
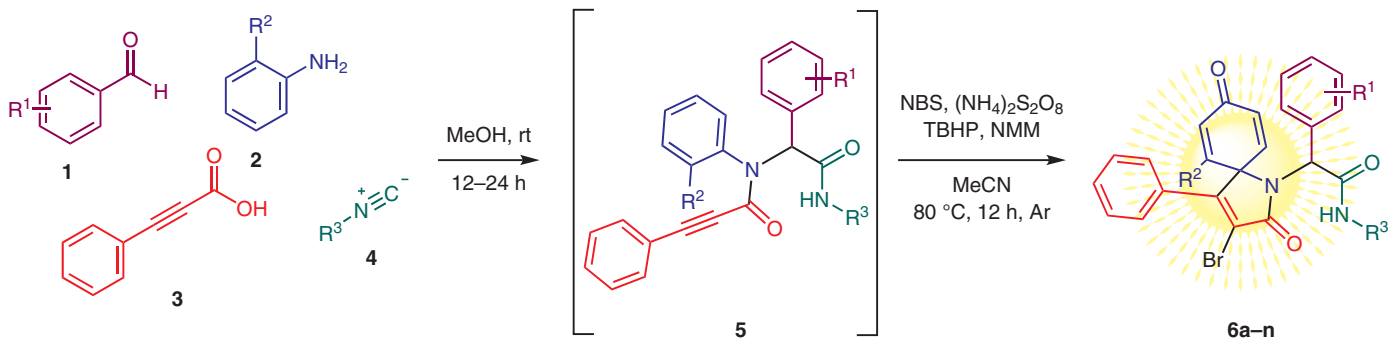

Scheme 1 Synthesis of 3-bromoazaspiro[4.5]trienones 6a-I via Ugi-4CR followed by ipso-bromocyclization 
Table 1 Optimization of the Reaction Conditions for the Synthesis of 3-Bromoazaspiro[4.5]trienone $\mathbf{6 a}$

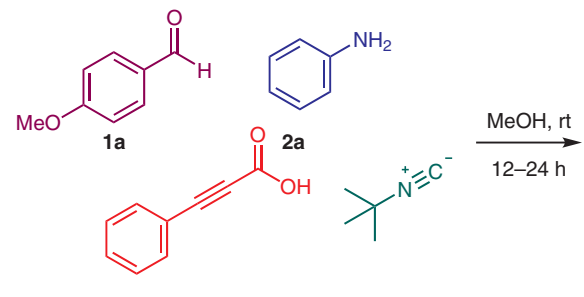

3
$4 a$

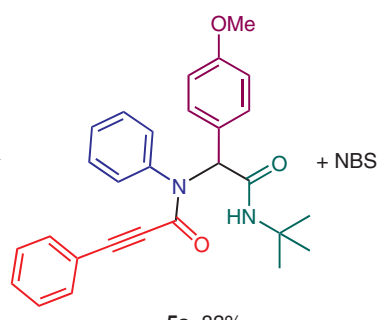

$5 a, 83 \%$

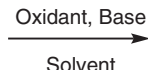

$80^{\circ} \mathrm{C}, 12 \mathrm{~h}, \mathrm{Ar}$

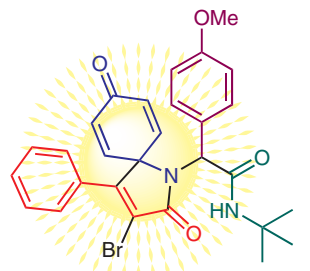

$6 a$

\begin{tabular}{|c|c|c|c|c|}
\hline Entry & Oxidant ( 3 equiv/ 5 equiv) & Base ( 0.5 equiv) & Solvent & Yield 6a $(\%)^{b}$ \\
\hline 1 & $\left(\mathrm{NH}_{4}\right)_{2} \mathrm{~S}_{2} \mathrm{O}_{8} / \mathrm{TBHP}$ & NMM & DMF & trace \\
\hline 2 & $\left(\mathrm{NH}_{4}\right)_{2} \mathrm{~S}_{2} \mathrm{O}_{8} / \mathrm{TBHP}$ & NMM & EtOH & 20 \\
\hline 3 & $\left(\mathrm{NH}_{4}\right)_{2} \mathrm{~S}_{2} \mathrm{O}_{8} / \mathrm{TBHP}$ & NMM & DCE & 20 \\
\hline 4 & $\left(\mathrm{NH}_{4}\right)_{2} \mathrm{~S}_{2} \mathrm{O}_{8} / \mathrm{TBHP}$ & NMM & toluene & 15 \\
\hline 5 & $\left(\mathrm{NH}_{4}\right)_{2} \mathrm{~S}_{2} \mathrm{O}_{8} / \mathrm{TBHP}$ & NMM & $\mathrm{H}_{2} \mathrm{O}$ & trace \\
\hline 6 & $\left(\mathrm{NH}_{4}\right)_{2} \mathrm{~S}_{2} \mathrm{O}_{8} / \mathrm{TBHP}$ & NMM & $\mathrm{H}_{2} \mathrm{O} / \mathrm{MeCN}$ & 25 \\
\hline 7 & $\left(\mathrm{NH}_{4}\right)_{2} \mathrm{~S}_{2} \mathrm{O}_{8} / \mathrm{TBHP}$ & NMM & $\mathrm{MeCN}$ & 88 \\
\hline 8 & $\left(\mathrm{NH}_{4}\right)_{2} \mathrm{~S}_{2} \mathrm{O}_{8} / \mathrm{TBHP}$ & NMP & $\mathrm{MeCN}$ & 75 \\
\hline 9 & $\left(\mathrm{NH}_{4}\right)_{2} \mathrm{~S}_{2} \mathrm{O}_{8} / \mathrm{TBHP}$ & TEA & $\mathrm{MeCN}$ & 40 \\
\hline 10 & $\left(\mathrm{NH}_{4}\right)_{2} \mathrm{~S}_{2} \mathrm{O}_{8} / \mathrm{TBHP}$ & $\mathrm{DBU}$ & $\mathrm{MeCN}$ & 59 \\
\hline 11 & $\left(\mathrm{NH}_{4}\right)_{2} \mathrm{~S}_{2} \mathrm{O}_{8} / \mathrm{TBHP}$ & DIEA & $\mathrm{MeCN}$ & 63 \\
\hline 12 & $\left(\mathrm{NH}_{4}\right)_{2} \mathrm{~S}_{2} \mathrm{O}_{8} /$ DTBP & NMM & $\mathrm{MeCN}$ & 23 \\
\hline 13 & $\left(\mathrm{NH}_{4}\right)_{2} \mathrm{~S}_{2} \mathrm{O}_{8} / \mathrm{BPO}$ & NMM & $\mathrm{MeCN}$ & 20 \\
\hline 14 & $\left(\mathrm{NH}_{4}\right)_{2} \mathrm{~S}_{2} \mathrm{O}_{8} / m-\mathrm{CPBA}$ & NMM & $\mathrm{MeCN}$ & 57 \\
\hline 15 & $\left(\mathrm{NH}_{4}\right)_{2} \mathrm{~S}_{2} \mathrm{O}_{8} / \mathrm{TBHP}$ & NMM & $\mathrm{MeCN}$ & $41^{c}$ \\
\hline
\end{tabular}

a Optimal reaction condition: 5 a (1 equiv), NBS (1.5 equiv), $\left(\mathrm{NH}_{4}\right)_{2} \mathrm{~S}_{2} \mathrm{O}_{8}$ (3 equiv), TBHP (5 equiv), and NMM (0.5 equiv) in MeCN (2 mL) at $80{ }^{\circ} \mathrm{C}$ under argon atmosphere for $12 \mathrm{~h}$.

b Isolated yield.

c This reaction was performed at room temperature.

tra of compounds exhibited characteristic signals at $\delta=$ 165.6, 166.9, $184.0 \mathrm{ppm}$ associated with the carbonyls of the amidic moieties and unsaturated ketone.

Initially, the effect of ring substituents on the aromatic aldehydes 6a-f was examined. Both electron-donating and electron-withdrawing substituents on the phenyl ring were tolerated as outlined (Scheme 2 ). With regard to the isocyanide component, tert-butyl isocyanide furnished the desired products in better yields than cyclohexyl isocyanide. A survey of aniline derivatives with differing substitution pat- terns revealed that aniline derivatives bearing substituents at the ortho-position were consistent with the optimal conditions, providing $6 \mathrm{~m}$ and $\mathbf{6 n}$ in moderate to good yields.

Treatment of para-halogen-substituted anilines with 4chlorobenzaldehyde (1b), phenylpropiolic acid (3), and tertbutyl isocyanide (4a) delivered product $\mathbf{6 b}$, but he reaction did not proceed at all when the aniline ring contained a para-nitro group (Scheme 3). It is noteworthy that, in all cases where yields were lower than average, the reaction was inefficient at the ipso-bromocyclization step, with a complex mixture of products being obtained. 
<smiles>[R]c1ccccc1N</smiles><smiles>COc1ccc(C(C(=O)NC(C)(C)C)N2C(=O)C(Br)=C(c3ccccc3)C23C=CC(=O)C=C3)cc1</smiles>

6a (67\%)<smiles>CC(C)(C)NC(=O)C(c1ccc(F)cc1)N1C(=O)C(Br)=C(c2ccccc2)C12C=CC(=O)C=C2</smiles>

$6 e(50 \%)$<smiles>O=C1C=CC2(C=C1)C(c1ccccc1)=C(Br)C(=O)N2C(C(=O)NC1CCCCC1)c1ccc([N+](=O)[O-])cc1</smiles>

6i $(49 \%)$

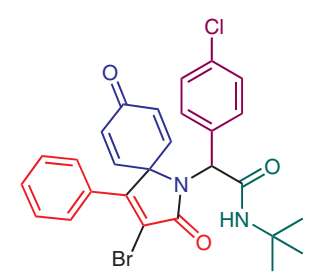

6b $(69 \%)$

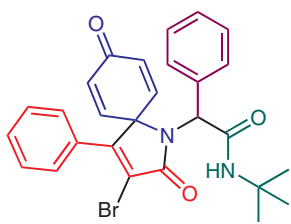

$6 f(73 \%)$

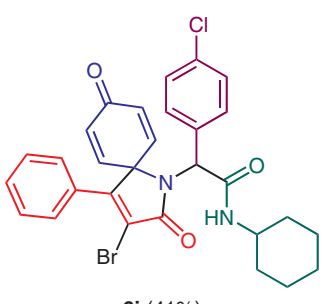

6j (41\%)

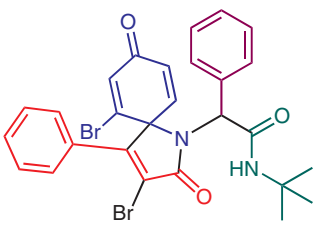

$6 \mathrm{~m}(38 \%)$

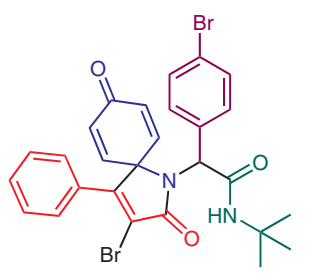

6c $(61 \%)$<smiles>O=C1C=CC2(C=C1)C(c1ccccc1)=C(Br)C(=O)N2C(C(=O)NC1CCCCC1)c1ccccc1</smiles>

$6 \mathrm{~g}(39 \%)$

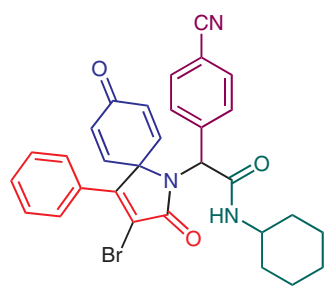

$6 k(20 \%)$

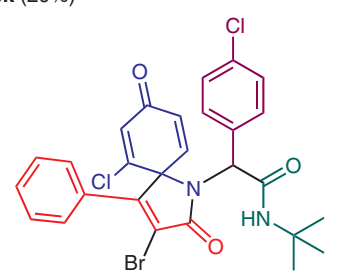

6n $(64 \%)$

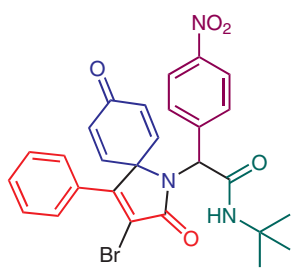

6d (60\%)

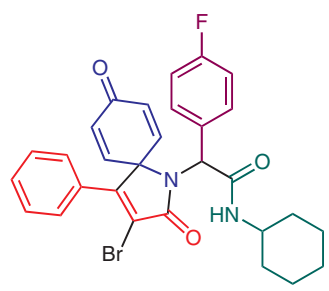

6h (48\%)

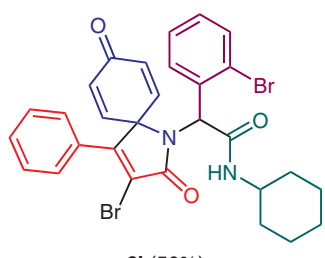

6I (50\%)

Scheme 2 Substrate scope for the synthesis of fully functionalized 3-bromoazaspiro[4.5]trienones $\mathbf{6 a - n}$<smiles>[C-]#[N+]C(C)(C)CCO</smiles>

$\mathrm{R}^{2}=\mathrm{Cl}, \mathrm{Br}$<smiles>CC(C)(C)NC(=O)N(C(=O)C#Cc1ccccc1)c1ccc(Br)cc1</smiles>

NBS, $\left(\mathrm{NH}_{4}\right)_{2} \mathrm{~S}_{2} \mathrm{O}_{8}$ TBHP, NMM $\mathrm{MeCN}$
$80^{\circ} \mathrm{C}, 12 \mathrm{~h}, \mathrm{Ar}$

$6 b=42 \%\left(R^{2}=C l\right)$

$\mathbf{6 b}=\operatorname{trace}\left(\mathrm{R}^{2}=\mathrm{Br}\right)$

Scheme 3 The effect of halogen substituents on the aniline ring at the para-position 
Crude products were purified on a silica gel column (EtOAc/n-hexane, 1:5) and the purified compounds were fully characterized by IR, ${ }^{1} \mathrm{H}$ NMR, ${ }^{13} \mathrm{C}$ NMR spectroscopy and HRMS analysis. In addition, in the case $\mathbf{6 a}$, the structure was confirmed by single-crystal X-ray diffraction analysis (Figure 3).

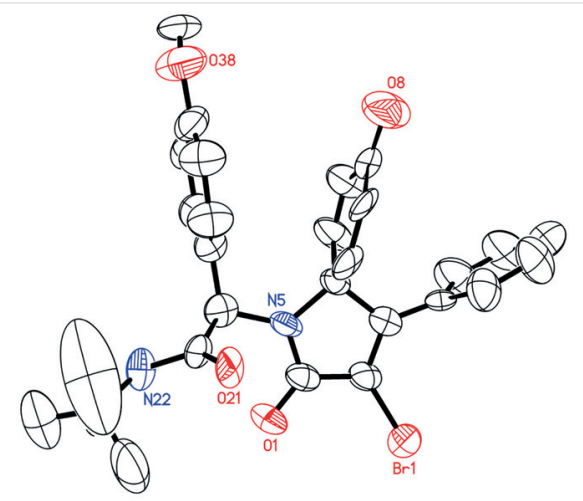

Figure 3 ORTEP view of compound $\mathbf{6 a}$
The proposed reaction mechanism involves the formation of vinyl radical $\mathbf{I}$ through the addition of a bromo radical generated from NBS and $\left(\mathrm{NH}_{4}\right)_{2} \mathrm{~S}_{2} \mathrm{O}_{8}$ to the alkyne group of the Ugi product. Subsequent intramolecular radical cyclization yields the intermediate II. Trapping of the radical intermediate II by the tert-butylperoxy radical generated from TBHP, and then elimination of tert-butyl alcohol provides the desired product (Scheme 4).

In conclusion, we have prepared a diverse array of fully functionalized 3-bromoazaspiro[4.5]trienones through Ugi-4CR followed by ipso-bromocyclization. Considering the biological importance of azaspiro[4.5]trienones and the potential utility of the bromo organic compounds to partake in further modifications, these compounds can be further exploited in the synthesis of lead compounds in medicinal chemistry.

\section{Funding Information}

We would like to thank the Iran National Science Foundation (INSF, Grant No. 96003234) and the National Institute for Medical Research Development (NIMAD, Grant No. 963388) for their financial support.<smiles>CNC(=O)C(c1ccccc1)N(C(=O)/C=C/c1ccccc1)c1ccccc1</smiles>

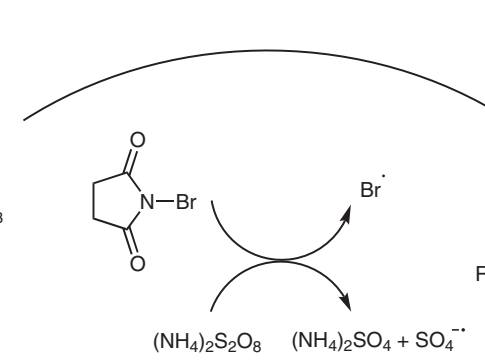<smiles>Cc1ccc(N(C(=O)C(Br)c2ccccc2)C(C(=O)NCCc2ccccc2)c2ccccc2)cc1</smiles>

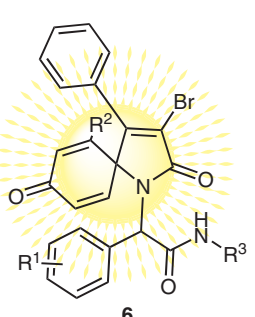<smiles>CC(CO)CC(C)CC(=O)O</smiles>

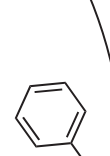<smiles>CCCC</smiles><smiles>CCC1(c2ccccc2)C(c2ccccc2)=C(Br)C(=O)N1C(C(=O)NC)c1ccccc1</smiles>

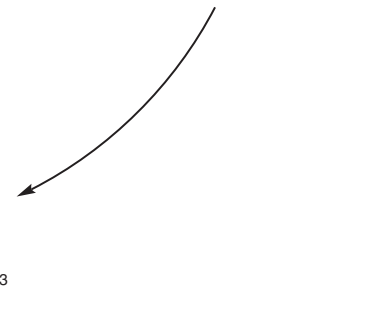

Scheme 4 The proposed reaction mechanism for the synthesis of 3-bromoazaspiro[4.5]trienones 6a-I 


\section{Supporting Information}

Supporting information for this article is available online at https://doi.org/10.1055/s-0037-1610205.

\section{References}

(1) Zheng, Y.; Tice, C. M.; Singh, S. B. Bioorg. Med. Chem. Lett. 2014, $24,3673$.

(2) Knox, C.; Law, V.; Jewison, T.; Liu, P.; Ly, S.; Frolkis, A.; Pon, A.; Banco, K.; Mak, C.; Neveu, V. Nucleic Acids Res. 2010, 39, D1035.

(3) (a) Li, M.; Song, R. J.; Li, J. H. Chin. J. Chem. 2017, 35, 299. (b) Jia, M. Q.; You, S. L. Chem. Commun. 2012, 6363. (c) Ouyang, X. H.; Song, R. J.; Liu, B.; Li, J. H. Chem. Commun. 2016, 2573.

(4) (a) Tang, B. X.; Zhang, Y. H.; Song, R. J.; Tang, D. J.; Deng, G. B.; Wang, Z. Q.; Xie, Y. X.; Xia, Y. Z.; Li, H. J.; J. Org. Chem. 2012, 77, 2837. (b) Ouyang, X. H.; Song, R. J.; Li, Y.; Liu, B.; Li, J. H. J. Org. Chem. 2014, 79, 4582. (c) Wen, J.; Wei, W.; Xue, S.; Yang, D.; Lou, Y.; Gao, C.; Wang, H. J. Org. Chem. 2015, 80, 4966.

(5) (a) RajiReddy, C.; Ranjan, R.; Prajapati, S. K.; Warudikar, K. J. Org. Chem. 2017, 82, 6932. (b) He, Y.; Qiu, G. Org. Biomol. Chem. 2017, 15, 3485. (c) Aparece, M. D.; Vadola, P. A. Org. Lett. 2014, $16,6008$.

(6) (a) Zhou, Y.; Zhang, X.; Zhang, Y.; Ruan, L.; Zhang, J.; ZhangNegrerie, D.; Du, Y. Org. Lett. 2016, 19, 150. (b) Wei, W. T.; Song, R. J.; Ouyang, X. H.; Li, Y.; Li, H. B.; Li, J. H. Org. Chem. Front. 2014, 1, 484. (c) Song, R.; Xie, Y. Chin. J. Chem. 2017, 35, 280.

(7) (a) Qian, P. C.; Liu, Y.; Song, R. J.; Xiang, J. N.; Li, J. H. Synlett 2015, 26, 1213. (b) Cui, H.; Wei, W.; Yang, D.; Zhang, J.; Xu, Z.; Wen, J.; Wang, H. RSC Adv. 2015, 5, 84657.

(8) (a) Godoi, B.; Schumacher, R. F.; Zeni, G. Chem. Rev. 2011, 111, 2837. (b) Likhar, P. R.; Subhas, M. S.; Roy, S.; Kantam, M. L.; Sridhar, B.; Seth, R. K.; Biswas, S. Org. Biomol. Chem. 2009, 7, 85.

(9) Yugandhar, D.; Nayak, V. L.; Archana, S.; Shekar, K. C.; Srivastava, A. K. Eur. J. Med. Chem. 2015, 101, 348.

(10) Weinreb, S. M. Chem. Rev. 2006, 106, 2531.

(11) Dutta, S.; Abe, H.; Aoyagi, S.; Kibayashi, C.; Gates, K. S. J. Am. Chem. Soc. 2005, 127, 15004

(12) Abe, H.; Aoyagi, S.; Kibayashi, C. J. Am. Chem. Soc. 2000, 122, 4583.

(13) Qiu, G.; Liu, T.; Ding, Q. Org. Chem. Front. 2016, 3, 510.

(14) Yugandhar, D.; Kuriakose, S.; Nanubolu, J. B.; Srivastava, A. K. Org. Lett. 2016, 18, 1040.

(15) Yugandhar, D.; Srivastava, A. K. ACS Comb. Sci. 2015, 17, 474.

(16) Saikia, I.; Borah, A. J.; Phukan, P. Chem. Rev. 2016, 116, 6837; and references cited therein.

(17) Balalaie, S.; Shamakli, M.; Nikbakht, A.; Alavijeh, N. S.; Rominger, F.; Rostamizadeh, S.; Bijanzadeh, H. R. Org. Biomol. Chem. 2017, 15, 5737.

(18) Sequential U4-CR/ipso-Bromocyclization to Synthesize Compounds 6a-o; General Procedure

To a solution of aldehyde $\mathbf{1 a}(1 \mathrm{mmol})$ in methanol $(5 \mathrm{~mL})$ was added aniline 2a $(1 \mathrm{mmol})$, and the reaction mixture was stirred at room temperature for $2 \mathrm{~h}$. Then, phenylpropiolic acid 3a $(1 \mathrm{mmol})$ was added and stirring was continued for $15 \mathrm{~min}$, followed by addition of isocyanide $4 \mathrm{a}(1 \mathrm{mmol})$; the solution was then stirred for $24 \mathrm{~h}$ at room temperature. The solvent was removed under reduced pressure and $\mathrm{MeCN}(10 \mathrm{~mL})$ was added to the residue. $\mathrm{N}$-Bromosuccinimide $(1.5 \mathrm{mmol}),\left(\mathrm{NH}_{4}\right)_{2} \mathrm{~S}_{2} \mathrm{O}_{8}(3$ $\mathrm{mmol})$, TBHP $(5 \mathrm{mmol})$ and NMM $(0.5 \mathrm{mmol})$ were added and the reaction mixture was stirred at $80^{\circ} \mathrm{C}$ for $12 \mathrm{~h}$ under an argon atmosphere. The progress of the reaction was monitored using TLC (n-hexane-EtOAc, 5:1). The resulting reaction mixture was concentrated under reduced pressure and the residue was purified by column chromatography (silica gel, appropriate mixture of $n$-hexane/ethyl acetate) to afford $\mathbf{6 a}$.

2-(3-Bromo-2,8-dioxo-4-phenyl-1-azaspiro[4.5]deca-3,6,9trien-1-yl)- $\mathrm{N}$-(tert-butyl)-2-(4-methoxyphenyl)acetamide (6a) Yield: $357 \mathrm{mg}$ (67\%); colorless solid; m.p. $240-242{ }^{\circ} \mathrm{C} ;{ }^{1} \mathrm{H}$ NMR $\left(\mathrm{CDCl}_{3}, 300 \mathrm{MHz}\right): \delta==1.30(\mathrm{~s}, 9 \mathrm{H}, 3 \mathrm{Me}), 3.79\left(\mathrm{~s}, 3 \mathrm{H},-\mathrm{OCH}_{3}\right)$, $4.77\left(\mathrm{~s}, 1 \mathrm{H}, \mathrm{C}\left(\mathrm{sp}^{3}\right)-\mathrm{H}\right), 5.50(\mathrm{~s}, 1 \mathrm{H}, \mathrm{N}-\mathrm{H}), 6.21(\mathrm{dd}, J=9.9,1.8 \mathrm{~Hz}$, $1 \mathrm{H},=\mathrm{CH}), 6.24(\mathrm{dd}, J=9.9,1.8 \mathrm{~Hz}, 1 \mathrm{H},=\mathrm{CH}), 6.53(\mathrm{dd}, J=9.9$, $3.1 \mathrm{~Hz}, 1 \mathrm{H},=\mathrm{CH}), 6.70(\mathrm{dd}, J=9.9,3.1 \mathrm{~Hz}, 1 \mathrm{H},=\mathrm{CH}), 6.83(\mathrm{~d}, J=$ $8.7 \mathrm{~Hz}, 2 \mathrm{H}, \mathrm{H}-\mathrm{Ar}$ ), 7.20-7.28 (m, $2 \mathrm{H}, \mathrm{H}-\mathrm{Ar}), 7.30-7.34$ (m, $3 \mathrm{H}$, $\mathrm{H}-\mathrm{Ar}), 7.36$ (d, $J=8.7 \mathrm{~Hz}, 2 \mathrm{H}, \mathrm{H}-\mathrm{Ar}) \cdot{ }^{13} \mathrm{C}$ NMR $\left(75 \mathrm{MHz} \mathrm{CDCl}_{3}\right.$ ): $\delta=28.5,51.9,55.3,62.1,69.5,114.3,120.1,127.1,127.8,128.5$, 130.0, 130.1, 131.0, 132.1, 132.2, 144.4, 152.3, 160.2, 165.6, 166.9, 184.0. HRMS (ESI): $\mathrm{m} / z[\mathrm{M}+\mathrm{H}]^{+}$calcd. for $\mathrm{C}_{28} \mathrm{H}_{28}{ }^{79} \mathrm{BrN}_{2} \mathrm{O}_{4}$ : 535.1227; found: 535.1232; $\mathrm{m} / z$ [M+Na] ${ }^{+}$calcd. for $\mathrm{C}_{28} \mathrm{H}_{27}{ }^{79} \mathrm{Br}-$ $\mathrm{N}_{2} \mathrm{NaO}_{4}$ : 557.1046; found: 557.1050; $\mathrm{m} / z[\mathrm{M}+\mathrm{K}]^{+}$calcd. for $\mathrm{C}_{28} \mathrm{H}_{27}{ }^{79} \mathrm{BrKN}_{2} \mathrm{O}_{4}$ : 573.0786; found: 573.0791; m/z [2 M+H] $]^{+}$ calcd. for $\mathrm{C}_{56} \mathrm{H}_{55}{ }^{79} \mathrm{Br}_{2} \mathrm{~N}_{4} \mathrm{O}_{8}$ : 1069.2381; found: $1069.2394 ; \mathrm{m} / \mathrm{z}$ [2 M+Na] ${ }^{+}$calcd. for $\mathrm{C}_{56} \mathrm{H}_{54}{ }^{79} \mathrm{Br}_{2} \mathrm{~N}_{4} \mathrm{NaO}_{8}$ : 1091.2201; found: 1091.2213; $\mathrm{m} / \mathrm{z} \quad[2 \mathrm{M}+\mathrm{K}]^{+}$calcd. for $\mathrm{C}_{56} \mathrm{H}_{54}{ }^{79} \mathrm{Br}_{2} \mathrm{KN}_{4} \mathrm{O}_{8}$ : 1107.1940; found: 1107.1952 . IR: $1663,1708,3316 \mathrm{~cm}^{-1}$.Crystal used for X-ray crystallographic analysis: colorless needle, dimensions $0.64 \times 0.05 \times 0.04 \mathrm{~mm}$. Crystal system: trigonal; space group: R3c; $Z=18 ; a=36.587(8) \AA, b=36.587(8) \AA, c=$ $9.942(2) \AA, \alpha=90 \mathrm{deg}, \beta=90 \mathrm{deg}, \gamma=120 \mathrm{deg} ; V=11525(6) \AA^{3}$; rho $=1.389 \mathrm{~g} / \mathrm{cm}^{3} ; T=200(2) \mathrm{K}$; Theta $_{\max }=17.402 \mathrm{deg}$; Radiation Mo K $\alpha ; \lambda=0.71073 \AA$; 0.5 deg omega-scans with CCD area detector, covering the asymmetric unit in reciprocal space with a mean redundancy of 23.1 and a completeness of $99.8 \%$ to a resolution of $1.19 \AA \AA$ A. 19242 Reflections measured, 1586 unique $(\mathrm{R}(\mathrm{int})=0.1093), 1419$ observed $(\mathrm{I}>2 \sigma(\mathrm{I}))$. Intensities were corrected for Lorentz and polarization effects, an empirical scaling and absorption correction was applied using SADABS based on the Laue symmetry of the reciprocal space, $\mathrm{mu}=1.64 \mathrm{~mm}^{-1}$, $T_{\min }=0.67, T_{\max }=0.95$, structure refined against $\mathrm{F}^{2}$ with a fullmatrix least-squares algorithm using the SHELXL-2016/6 (Sheldrick, 2016) software. ${ }^{19} 316$ Parameters were refined, hydrogen atoms were treated using appropriate riding models. Flack absolute structure parameter $0.077(16)$, goodness of fit 1.15 for observed reflections, final residual values $\mathrm{R} 1(\mathrm{~F})=0.071, \mathrm{wR}\left(\mathrm{F}^{2}\right)=$ 0.145 for observed reflections, residual electron density -0.30 to $0.38 \mathrm{e}^{-3}$. CCDC 1587337 contains the supplementary crystallographic data for this paper. These data can be obtained free of charge from The Cambridge Crystallographic Data Centre via www.ccdc.cam.ac.uk/data-request/cif.

2-(3-Bromo-2,8-dioxo-4-phenyl-1-azaspiro[4.5]deca-3,6,9trien-1-yl)-2-(4-bromophenyl)- $\mathrm{N}$-(tert-butyl)acetamide (6c) Yield: $355 \mathrm{mg}$ (61\%); colorless solid; m.p. $250-252{ }^{\circ} \mathrm{C}$; ${ }^{1} \mathrm{H}$ NMR $\left(\mathrm{CDCl}_{3}, 300 \mathrm{MHz}\right): \delta=1.29(\mathrm{~s}, 9 \mathrm{H}, 3 \mathrm{Me}), 4.60\left(\mathrm{~s}, 1 \mathrm{H}, \mathrm{C}\left(\mathrm{sp}^{3}\right)-\mathrm{H}\right)$, $5.51(\mathrm{~s}, 1 \mathrm{H}, \mathrm{N}-\mathrm{H}), 6.25(\mathrm{dd}, J=9.9,1.8 \mathrm{~Hz}, 1 \mathrm{H},=\mathrm{CH}), 6.37(\mathrm{dd}, J=$ 9.9, $1.8 \mathrm{~Hz}, 1 \mathrm{H},=\mathrm{CH}), 6.44(\mathrm{dd}, J=10.0,3.0 \mathrm{~Hz}, 1 \mathrm{H},=\mathrm{CH}), 6.81$ (dd, $J=10.0,3.0 \mathrm{~Hz}, 1 \mathrm{H},=\mathrm{CH}), 7.24-7.28(\mathrm{~m}, 2 \mathrm{H}, \mathrm{H}-\mathrm{Ar}), 7.31-$ 7.36 (m, $5 \mathrm{H}, \mathrm{H}-\mathrm{Ar}$ ), 7.49 (d, $J=8.4 \mathrm{~Hz}, 2 \mathrm{H}, \mathrm{H}-\mathrm{Ar}) .{ }^{13} \mathrm{C}$ NMR $\left(75 \mathrm{MHz}, \mathrm{CDCl}_{3}\right): \delta=28.5,52.2,62.3,69.6,119.9,123.5,127.8$, $128.7,130.0,130.2,130.9,132.4,132.6,133.0,134.4,143.8$ 144.1, 152.5, 165.8, 166.0, 183.7. HRMS (ESI): $\mathrm{m} / z[\mathrm{M}+\mathrm{H}]^{+}$calcd. for $\mathrm{C}_{27} \mathrm{H}_{25}{ }^{79} \mathrm{Br}_{2} \mathrm{~N}_{2} \mathrm{O}_{3}$ : 583.0226; found 583.0233; $\mathrm{m} / \mathrm{z}[\mathrm{M}+\mathrm{Na}]^{+}$ 
calcd. for $\mathrm{C}_{27} \mathrm{H}_{24}{ }^{79} \mathrm{Br}_{2} \mathrm{~N}_{2} \mathrm{NaO}_{3}$ : 605.0046; found: 605.0051; $\mathrm{m} / \mathrm{z}$ $[\mathrm{M}+\mathrm{K}]^{+}$calcd. for $\mathrm{C}_{27} \mathrm{H}_{24}{ }^{79} \mathrm{Br}_{2} \mathrm{KN}_{2} \mathrm{O}_{3}$ : 620.9785; found: 620.9794 . IR: $1621,1692,1709,3417 \mathrm{~cm}^{-1}$.

2-(3-Bromo-2,8-dioxo-4-phenyl-1-azaspiro[4.5]deca-3,6,9trien-1-yl)- $\mathrm{N}$-cyclohexyl-2-(4-fluorophenyl)acetamide (6h) Yield: $263 \mathrm{mg}$ (48\%); colorless solid; m.p. $268-269{ }^{\circ} \mathrm{C} ;{ }^{1} \mathrm{H}$ NMR $\left(\mathrm{CDCl}_{3}, 300 \mathrm{MHz}\right): \delta=1.015-1.14(\mathrm{~m}, 3 \mathrm{H}, \mathrm{H}-\mathrm{cyc}), 1.22-1.32(\mathrm{~m}$, 2 H, H-cyc), 1.57 (s, 3 H, H-cyc), 1.76-1.93 (m, 2 H, H-cyc), 3.76$3.78(\mathrm{~m}, 1 \mathrm{H}, \mathrm{H}-\mathrm{cyc}), 4.83\left(\mathrm{~s}, 1 \mathrm{H}, \mathrm{C}\left(\mathrm{sp}^{3}\right)-\mathrm{H}\right), 5.65(\mathrm{~d}, J=7.8 \mathrm{~Hz}$, $1 \mathrm{H}, \mathrm{N}-\mathrm{H}), 6.27(\mathrm{~d}, J=9.9 \mathrm{~Hz}, 1 \mathrm{H},=\mathrm{CH}), 6.32(\mathrm{~d}, J=9.9 \mathrm{~Hz}, 1 \mathrm{H}$, $=\mathrm{CH}), 6.50(\mathrm{dd}, J=9.9,2.4 \mathrm{~Hz}, 1 \mathrm{H},=\mathrm{CH}), 6.78(\mathrm{dd}, J=9.9,2.4 \mathrm{~Hz}$, $1 \mathrm{H},=\mathrm{CH}), 7.04(\mathrm{t}, J=8.7 \mathrm{~Hz}, 2 \mathrm{H}, \mathrm{H}-\mathrm{Ar}), 7.27(\mathrm{~d}, J=7.2 \mathrm{~Hz}, 2 \mathrm{H}$, H-Ar), 7.35 (d, J = 7.2 Hz, $2 \mathrm{H}, \mathrm{H}-\mathrm{Ar}$ ), 7.40-7.65 (m, $3 \mathrm{H}, \mathrm{H}-\mathrm{Ar}$ ). ${ }^{13} \mathrm{C}$ NMR ( $75 \mathrm{MHz}, \mathrm{CDCl}_{3}$ ): $\delta=24.5,24.6,25.3,32.5,32.7,49.1$, $61.1,69.6,116.2\left(\mathrm{~d},{ }^{2} J_{\mathrm{C}-\mathrm{F}}=21.0 \mathrm{~Hz}\right), 119.8,127.8,128.4,128.6$, $130.2\left(\mathrm{~d},{ }^{3} J_{\mathrm{C}-\mathrm{F}}=7.8 \mathrm{~Hz}\right), 131.0,131.4,131.5,132.6,132.7,143.9$, 144.2, 152.6, 161.4, 165.9, 166.4, 183.8. HRMS (ESI): $m / z[M+H]^{+}$ calcd. for $\mathrm{C}_{29} \mathrm{H}_{27}{ }^{79} \mathrm{BrFN}_{2} \mathrm{O}_{3}$ : 549.1184; found: $549.1187 ; \mathrm{m} / \mathrm{z}$ $[\mathrm{M}+\mathrm{K}]^{+}$calcd. for $\mathrm{C}_{29} \mathrm{H}_{26}{ }^{79} \mathrm{BrFKN}_{2} \mathrm{O}_{3}$ : 587.0742; found: 587.0746. IR: $1659,1713,3254 \mathrm{~cm}^{-1}$.

$\mathrm{N}$-(tert-butyl)-2-(3,6-dibromo-2,8-dioxo-4-phenyl-1azaspiro[4.5]deca-3,6,9-trien-1-yl)-2-phenylacetamide (6m) Yield: $222 \mathrm{mg}$ (38\%); yellow solid; m.p. $238-239{ }^{\circ} \mathrm{C} ;{ }^{1} \mathrm{H}$ NMR $\left(\mathrm{CDCl}_{3}, 300 \mathrm{MHz}\right): \delta=1.36(\mathrm{~s}, 9 \mathrm{H}, 3 \mathrm{Me}), 5.38\left(\mathrm{~s}, 1 \mathrm{H}, \mathrm{C}\left(\mathrm{sp}^{3}\right)-\mathrm{H}\right)$, 5.97 (br. s, $1 \mathrm{H}, \mathrm{N}-\mathrm{H}), 6.26$ (dd, $J=9.9,1.6 \mathrm{~Hz}, 1 \mathrm{H},=\mathrm{CH}), 6.28$ (d, $J=1.6 \mathrm{~Hz}, 1 \mathrm{H},=\mathrm{CH}), 7.20-7.42(\mathrm{~m}, 11 \mathrm{H}, \mathrm{H}-\mathrm{Ar},=\mathrm{CH}) .{ }^{13} \mathrm{C} \mathrm{NMR}$ $\left(75 \mathrm{MHz}, \mathrm{CDCl}_{3}\right): \delta=28.6,52.0,63.3,72.7,121.0,128.0,128.2$, 128.6, 129.1, 129.7, 130.2, 130.6, 130.9, 131.7, 135.9, 142.8, 144.0, 152.5, 166.6, 167.2, 181.9. MS (ESI): $\mathrm{m} / z[\mathrm{M}+\mathrm{H}]^{+}$found for $\mathrm{C}_{27} \mathrm{H}_{24}{ }^{79} \mathrm{Br}_{2} \mathrm{~N}_{2} \mathrm{O}_{3}$ : 582.6; $\mathrm{m} / z[\mathrm{M}+\mathrm{H}]^{+}$found for $\mathrm{C}_{27} \mathrm{H}_{24}{ }^{81} \mathrm{Br}_{2} \mathrm{~N}_{2} \mathrm{O}_{3}$ : 584.6; IR: $1713,3322 \mathrm{~cm}^{-1}$.

(19) (a) Sheldrick, G. M. Bruker Analytical X-ray-Division: Madison, Wisconsin 2014. (b) Sheldrick, G. M. Acta Crystallogr., Sect. C: Cryst. Struct. Commun. 2015, 71, 3. 